Pacific

Journal of

Mathematics

COMPACT HYPERSURFACES IN A UNIT SPHERE WITH INFINITE FUNDAMENTAL GROUP

QING-Ming Cheng

Volume $212 \quad$ No. 1

November 2003 


\title{
COMPACT HYPERSURFACES IN A UNIT SPHERE WITH INFINITE FUNDAMENTAL GROUP
}

\author{
Qing-Ming Cheng
}

\begin{abstract}
It is our purpose to study curvature structures of compact hypersurfaces in the unit sphere $S^{n+1}(1)$. We proved that the Riemannian product $S^{1}\left(\sqrt{1-c^{2}}\right) \times S^{n-1}(c)$ is the only compact hypersurfaces in $S^{n+1}(1)$ with infinite fundamental group, which satisfy $r \geq \frac{n-2}{n-1}$ and $S \leq(n-1) \frac{n(r-1)+2}{n-2}+$ $\frac{n-2}{n(r-1)+2}$, where $n(n-1) r$ is the scalar curvature of hypersurfaces and $c^{2}=\frac{n-2}{n r}$. In particular, we obtained that the Riemannian product $S^{1}\left(\sqrt{1-c^{2}}\right) \times S^{n-1}(c)$ is the only compact hypersurfaces with infinite fundamental group in $S^{n+1}(1)$ if the sectional curvatures are nonnegative.
\end{abstract}

\section{Introduction.}

Let $M$ be an $n$-dimensional hypersurface in a unit sphere $S^{n+1}(1)$ of dimension $n+1$. It is well-known that the investigation on curvature structures of compact hypersurfaces is important and interesting. In 1977, S.Y. Cheng and Yau [4] studied compact hypersurfaces with constant scalar curvature in the unit sphere $S^{n+1}(1)$. They proved that let $M$ be an $n$-dimensional compact hypersurface with constant scalar curvature $n(n-1) r$, if $r \geq 1$ and the sectional curvatures of $M$ are nonnegative, then $M$ is isometric to the totally umbilical hypersurface $S^{n}(c)$ or the Riemannian product $S^{k}\left(c_{1}\right) \times S^{n-k}\left(c_{2}\right)$ $1 \leq k \leq n-1$, where $S^{k}(c)$ denote the sphere of radius $c$. In order to prove this theorem, they introduced a differential operator $\square$ defined by

$$
\square f=\sum_{i, j=1}^{n}\left(n H \delta_{i j}-h_{i j}\right) \nabla_{i} \nabla_{j} f,
$$

for any $C^{2}$-function $f$ on $M$. Where $h_{i j}$ and $H$ are components of the second fundamental form and the mean curvature of $M$, respectively. We should notice the following:

(1) The differential operator $\square$ is self-adjoint.

(2) The differential operator $\square$ is degenerate elliptic if $r \geq 1$.

Therefore, in order to prove their theorem, they must make use of the properties that the differential operator $\square$ is self-adjoint and degenerate elliptic. 
And in order to obtain the estimate $\sum_{i, j, k=1}^{n} h_{i j k}^{2} \geq n^{2}|\operatorname{grad} H|^{2}$, which is very important in the proof of their theorem, the condition of $r \geq 1$ and the assumption of constant scalar curvature is essential. Where $h_{i j k}$ 's are components of the covariant differentiation of the second fundamental form. Hence, the condition $r \geq 1$ and the assumption of constant scalar curvature play an essential role in the proof of their theorem. Further, by making use of the similar method which was used by Nakagawa and the author in [3] and the differential operator introduced by S.Y. Cheng and Yau, Li [5] proved that let $M$ be an $n$-dimensional compact hypersurface with constant scalar curvature $n(n-1) r$, if $r \geq 1$ and $S \leq(n-1) \frac{n(r-1)+2}{n-2}+\frac{n-2}{n(r-1)+2}$, then $M$ is isometric to either the totally umbilical hypersurface or the Riemannian product $S^{1}\left(\sqrt{1-c^{2}}\right) \times S^{n-1}(c)$ with $c^{2}=\frac{n-2}{n r} \leq \frac{n-2}{n}$, where $S$ is the squared norm of the second fundamental form of $M$. These properties that the differential operator $\square$ is self-adjoint and degenerate elliptic are indispensable in the proof of his theorem again. And the estimate $\sum_{i, j, k=1}^{n} h_{i j k}^{2} \geq n^{2}|\operatorname{grad} H|^{2}$ is also essential in the proof of his theorem.

On the other hand, for any $0<c<1$, by considering the standard immersions $S^{n-1}(c) \subset \mathbf{R}^{n}, S^{1}\left(\sqrt{1-c^{2}}\right) \subset \mathbf{R}^{2}$ and taking the Riemannian product immersion $S^{1}\left(\sqrt{1-c^{2}}\right) \times S^{n-1}(c) \hookrightarrow \mathbf{R}^{2} \times \mathbf{R}^{n}$, we obtain a compact hypersurface $S^{1}\left(\sqrt{1-c^{2}}\right) \times S^{n-1}(c)$ in $S^{n+1}(1)$ with constant scalar curvature $n(n-1) r$, where $r=\frac{n-2}{n c^{2}}>1-\frac{2}{n}$. Hence, some of Riemannian products $S^{1}\left(\sqrt{1-c^{2}}\right) \times S^{n-1}(c)$ do not appear in these results of S.Y. Cheng and Yau [4] and $\mathrm{Li}[\mathbf{5}]$. Moreover, Cheng [2] proved:

Theorem C (Cheng [2]). Let $M$ be an n-dimensional complete hypersurface with constant scalar curvature $n(n-1) r$ in $S^{n+1}(1)$. If $M$ has only two distinct principal curvatures one of which is simple, then, $r>1-\frac{2}{n}$ holds and $M$ is isometric to $S^{1}\left(\sqrt{1-c^{2}}\right) \times S^{n-1}(c)$ if $r \neq \frac{n-2}{n-1}$ and $S \geq$ $(n-1) \frac{n(r-1)+2}{n-2}+\frac{n-2}{n(r-1)+2}$, where $c^{2}=\frac{n-2}{n r}$.

From the assertion above, it is natural and interesting to generalize these results due to S.Y. Cheng and Yau [4] and Li [5] to the case $r>1-\frac{2}{n}$. That is, it is interesting to prove the following:

Problem 1 (cf. Cheng [2]). Let $M$ be an $n$-dimensional compact hypersurface with constant scalar curvature $n(n-1) r$ in $S^{n+1}(1)$. If $r>1-\frac{2}{n}$ and $S \leq(n-1) \frac{n(r-1)+2}{n-2}+\frac{n-2}{n(r-1)+2}$, then $M$ is isometric to the totally umbilical hypersurface or the Riemannian product $S^{1}\left(\sqrt{1-c^{2}}\right) \times S^{n-1}(c)$.

It is our purpose to try to solve this problem above. Since the problem seems to be a very hard problem, we shall try to solve it under a topological condition. It is known that $S^{1}\left(\sqrt{1-c^{2}}\right) \times S^{n-1}(c)$ has infinite fundamental 
group. Hence, we shall consider compact hypersurfaces with infinite fundamental group in the unit sphere $S^{n+1}(1)$. The following theorems will be proved.

Theorem 1. Let $M$ be an n-dimensional compact hypersurface with infinite fundamental group in $S^{n+1}(1)$. If $r \geq \frac{n-2}{n-1}$ and $S \leq(n-1) \frac{n(r-1)+2}{n-2}+$ $\frac{n-2}{n(r-1)+2}$, then $M$ is isometric to the Riemannian product $S^{1}\left(\sqrt{1-c^{2}}\right) \times$ $S^{n-1}(c)$, where $n(n-1) r$ is the scalar curvature of $M$ and $c^{2}=\frac{n-2}{n r}$.

Theorem 2. Let $M$ be an n-dimensional compact hypersurface with infinite fundamental group in $S^{n+1}(1)$. If the sectional curvatures are nonnegative, then $M$ is isometric to the Riemannian product $S^{1}\left(\sqrt{1-c^{2}}\right) \times S^{n-1}(c)$.

Remark. In our Theorems 1 and 2, we do not assume that the scalar curvature is constant. And in our Theorem 2, we do not assume any condition on scalar curvature.

\section{Proofs of theorems.}

Let $M$ be an $n$-dimensional hypersurface in a unit sphere $S^{n+1}(1)$ with constant scalar curvature $n(n-1) r$. We take a local orthonormal frame field $\left\{e_{1}, \ldots, e_{n+1}\right\}$ in $S^{n+1}(1)$, restricted to $M$, so that $e_{1}, \ldots, e_{n}$ are tangent to $M$. Let $\omega_{1}, \cdots, \omega_{n+1}$ denote the dual coframe fields in $S^{n+1}(1)$. We shall make use of the following convention on the ranges of indices:

$$
1 \leq A, B, C, \cdots, \leq n+1 ; \quad 1 \leq i, j, k, \cdots, \leq n .
$$

Then the structure equations of $S^{n+1}(1)$ are given by

$$
\begin{gathered}
d \omega_{A}=\sum_{B=1}^{n+1} \omega_{A B} \wedge \omega_{B}, \quad \omega_{A B}+\omega_{B A}=0 \\
d \omega_{A B}=\sum_{C=1}^{n+1} \omega_{A C} \wedge \omega_{C B}+\Omega_{A B}, \quad \Omega_{A B}=-\frac{1}{2} \sum_{C, D=1}^{n+1} R_{A B C D} \omega_{C} \wedge \omega_{D}, \\
R_{A B C D}=\left(\delta_{A C} \delta_{B D}-\delta_{A D} \delta_{B C}\right),
\end{gathered}
$$

where $\Omega_{A B}$ (resp. $R_{A B C D}$ ) denotes the curvature form (resp. the components of the curvature tensor) of $S^{n+1}(1)$. Then, in $M$,

$$
\omega_{n+1}=0 .
$$

It follows from Cartan's Lemma that

$$
\omega_{n+1 i}=\sum_{j} h_{i j} \omega_{j}, \quad h_{i j}=h_{j i} .
$$


The second fundamental form $\alpha$ and the mean curvature of $M$ are defined by

$$
\alpha=\sum_{i, j} h_{i j} \omega_{i} \omega_{j} e_{n+1} \quad \text { and } \quad n H=\sum_{i} h_{i i},
$$

respectively. $M$ is said to be totally umbilical if the $h_{i j}$ can be expressed as $h_{i j}=H \delta_{i j}$. The structure equations of $M$ are given by

$$
\begin{gathered}
d \omega_{i}=\sum_{k=1}^{n} \omega_{i k} \wedge \omega_{k}, \quad \omega_{i j}+\omega_{j i}=0 \\
d \omega_{i j}=\sum_{k=1}^{n} \omega_{i k} \wedge \omega_{k j}+\Omega_{i j}, \quad \Omega_{i j}=-\frac{1}{2} \sum_{k, l=1}^{n} R_{i j k l} \omega_{k} \wedge \omega_{l} \\
R_{i j k l}=\left(\delta_{i k} \delta_{j l}-\delta_{i l} \delta_{j k}\right)+\left(h_{i k} h_{j l}-h_{i l} h_{j k}\right)
\end{gathered}
$$

where $\Omega_{i j}$ (resp. $R_{i j k l}$ ) denotes the Riemannian curvature form (resp. the components of the Riemannian curvature tensor) of $M$. From the above equation, we have

$$
\begin{gathered}
R_{i j}=(n-1) \delta_{i j}+n H h_{i j}-\sum_{k=1}^{n} h_{i k} h_{k j}, \\
n(n-1) r=n(n-1)+n^{2} H^{2}-S,
\end{gathered}
$$

where $R_{i j}$ and $n(n-1) r$ are components of the Ricci curvature tensor and the scalar curvature of $M$, respectively, and $S=\sum_{i j=1}^{n} h_{i j}^{2}$ is the squared norm of the second fundamental form of $M$.

Proof of Theorem 1. Since $r \geq \frac{n-2}{n-1}$ and $S \leq(n-1) \frac{n(r-1)+2}{n-2}+\frac{n-2}{n(r-1)+2}$, we infer

$$
n+2(n-1)(r-1)-\frac{n-2}{n} S \geq 0 .
$$

In fact,

$$
\begin{aligned}
n+ & 2(n-1)(r-1)-\frac{n-2}{n} S \\
= & n+2(n-1)(r-1)-\frac{n-2}{n}\left\{(n-1) \frac{n(r-1)+2}{n-2}+\frac{n-2}{n(r-1)+2}\right\} \\
& +\frac{n-2}{n}\left\{(n-1) \frac{n(r-1)+2}{n-2}+\frac{n-2}{n(r-1)+2}-S\right\} \\
\geq & n+2(n-1)(r-1)-\frac{n-2}{n}\left\{(n-1) \frac{n(r-1)+2}{n-2}+\frac{n-2}{n(r-1)+2}\right\} \\
= & \frac{(n-2)^{2}}{n}+\frac{n-1}{n}\{n(r-1)+2\}-\frac{(n-2)^{2}}{n\{n(r-1)+2\}} .
\end{aligned}
$$


We consider function $f(t)=\frac{(n-2)^{2}}{n}+\frac{n-1}{n} t-\frac{(n-2)^{2}}{n t}$. By a direct computation, we have $f(t) \geq 0$ if $t \geq \frac{n-2}{n-1}$. Since $r \geq \frac{n-2}{n-1}$, we have $n(r-1)+2 \geq \frac{n-2}{n-1}$. Thus, we infer

$$
n+2(n-1)(r-1)-\frac{n-2}{n} S \geq 0 .
$$

Therefore, when $r \geq \frac{n-2}{n-1}$, we know that

$$
S \leq(n-1) \frac{n(r-1)+2}{n-2}+\frac{n-2}{n(r-1)+2}
$$

is equivalent to

$$
\begin{aligned}
& n+2(n-1)(r-1)-\frac{n-2}{n} S \\
& \geq \frac{n-2}{n} \sqrt{\{n(n-1)(r-1)+S\}\{S-n(r-1)\}} .
\end{aligned}
$$

Indeed, we can prove that

$$
S \leq(n-1) \frac{n(r-1)+2}{n-2}+\frac{n-2}{n(r-1)+2}
$$

holds if and only if

$$
\begin{aligned}
& \left\{n+2(n-1)(r-1)-\frac{n-2}{n} S\right\}^{2} \\
& \geq \frac{(n-2)^{2}}{n^{2}}\{n(n-1)(r-1)+S\}\{S-n(r-1)\} .
\end{aligned}
$$

Since (2.5) holds, we obtain that (2.7) is true.

From Gauss equation $n(n-1) r=n(n-1)+n^{2} H^{2}-S$, we conclude

$$
\{S-n(r-1)\}=\frac{n}{n-1}\left(S-n H^{2}\right) .
$$

Hence, from (2.7) and (2.8), we obtain

$$
n+2 n H^{2}-S \geq \frac{n-2}{\sqrt{n(n-1)}} \sqrt{n^{2} H^{2}\left(S-n H^{2}\right)} .
$$

For any point $p$ and any unit vector $\vec{u} \in T_{p} M$, we choose a local orthonormal frame field $\left\{e_{1}, \cdots, e_{n}\right\}$ such that $e_{n}=\vec{u}$, we have, from Gauss equation $(2.3)$,

$$
\operatorname{Ric}(\vec{u})=(n-1)+n H h_{n n}-\sum_{i=1}^{n} h_{i n}^{2} .
$$

Since

$$
\left(n H-h_{n n}\right)^{2}=\left(\sum_{i=1}^{n-1} h_{i i}\right)^{2} \leq(n-1) \sum_{i=1}^{n-1} h_{i i}^{2}
$$


we have

$$
n^{2} H^{2}-(n-1) \sum_{i=1}^{n} h_{i i}^{2}+n h_{n n}^{2}-2 n H h_{n n} \leq 0 .
$$

From $\sum_{i}\left(h_{i i}-H\right)=0$ and $\sum_{i=1}^{n}\left(h_{i i}-H\right)^{2}=\sum_{i=1}^{n} h_{i i}^{2}-n H^{2}$, we have, for any $i$,

$$
\begin{gathered}
\left(h_{i i}-H\right)^{2} \leq \frac{n-1}{n}\left(\sum_{i=1}^{n} h_{i i}^{2}-n H^{2}\right) . \\
0 \geq n\left(h_{n n}^{2}-n H h_{n n}\right)+(n-2) n H\left(h_{n n}-H\right) \\
+2(n-1) n H^{2}-(n-1) \sum_{i=1}^{n} h_{i i}^{2} \\
\geq n\left(h_{n n}^{2}-n H h_{n n}\right)-(n-2) n|H| \sqrt{\frac{n-1}{n}\left(\sum_{i=1}^{n} h_{i i}^{2}-n H^{2}\right)} \\
+2(n-1) n H^{2}-(n-1) \sum_{i=1}^{n} h_{i i}^{2},
\end{gathered}
$$

namely,

$$
\begin{aligned}
& \left(h_{n n}^{2}-n H h_{n n}\right) \\
& \leq(n-2)|H| \sqrt{\frac{n-1}{n}\left(\sum_{i=1}^{n} h_{i i}^{2}-n H^{2}\right)}-2(n-1) H^{2}+\frac{n-1}{n} \sum_{i=1}^{n} h_{i i}^{2} .
\end{aligned}
$$

From (2.10) and (2.11), we have

$$
\begin{aligned}
\operatorname{Ric}(\vec{u}) \geq & (n-1)-(n-2)|H| \sqrt{\frac{n-1}{n}\left(\sum_{i=1}^{n} h_{i i}^{2}-n H^{2}\right)} \\
& +2(n-1) H^{2}-\frac{n-1}{n} \sum_{i, j=1}^{n} h_{i j}^{2}
\end{aligned}
$$

because of $\frac{n-1}{n}>\frac{1}{2}$. Thus, we obtain, from the above inequality and $S=$ $\sum_{i, j=1}^{n} h_{i j}^{2}$,

(2.12) $\operatorname{Ric}(\vec{u}) \geq \frac{n-1}{n}\left\{n+2 n H^{2}-S-\frac{n-2}{\sqrt{n(n-1)}} \sqrt{n^{2} H^{2}\left(S-n H^{2}\right)}\right\}$.

From (2.9), we have $\operatorname{Ric}(\vec{u}) \geq 0$. In particular, from the assertions above, we know that if $S<(n-1) \frac{n(r-1)+2}{n-2}+\frac{n-2}{n(r-1)+2}$ holds, then $\operatorname{Ric}(\vec{u})>0$. Thus, if 
there exists point $p$ in $M$ such that $S<(n-1) \frac{n(r-1)+2}{n-2}+\frac{n-2}{n(r-1)+2}$, then, at the point $p$, the Ricci curvature is positive. From the following Lemma due to Aubin [1], we know that there exists a metric on $M$ such that the Ricci curvature is positive on $M$. According to Myers theorem, we know that the fundamental group is finite. This is impossible because $M$ has infinite fundamental group.

Lemma (cf. Aubin [1, p. 344]). If the Ricci curvature of a compact Riemannian manifold is nonnegative and positive at somewhere, then the manifold carries a metric with positive Ricci curvature.

Thus, we must have $S=(n-1) \frac{n(r-1)+2}{n-2}+\frac{n-2}{n(r-1)+2}$. And at each point, there exists a unit vector $\vec{u} \operatorname{such}$ that $\operatorname{Ric}(\vec{u})=0$. From the assertions above, we infer that these inequalities above are equalities. That is, we must have $h_{i j}=0$ if $i \neq j$ and $h_{11}=\cdots=h_{n-1 n-1}$,

$$
\left(h_{n n}-H\right)^{2}=\frac{n-1}{n}\left(\sum_{i=1}^{n} h_{i i}^{2}-n H^{2}\right)=\frac{n-1}{n}\left(S-n H^{2}\right)
$$

and

$$
\left(h_{11}-H\right)^{2}=\cdots=\left(h_{n-1 n-1}-H\right)^{2}=\frac{1}{n(n-1)}\left(S-n H^{2}\right) .
$$

Hence, we conclude that $M$ has only two distinct principal curvatures one of which is simple. Let $\left\{e_{1}, \cdots, e_{n}\right\}$ be a local orthonormal frame field such that $h_{i j}=\lambda_{i} \delta_{i j}$, where $\lambda_{i}$ 's are principal curvatures on $M$. Without loss of generality, we can assume $\mu=\lambda_{n}, \lambda=\lambda_{1}=\cdots=\lambda_{n-1}$. From Gauss Equation (2.2) and the definition of the Ricci curvature, we have $1+\mu \lambda=0$ because of $1+\lambda_{i} \lambda_{j}=1+\lambda^{2}>0$, for any $i, j=1, \cdots, n-1$. From (2.4), we have

$$
\mu=\frac{n(r-1)}{2 \lambda}-\frac{n-2}{2} \lambda .
$$

Hence $\lambda^{2}=\frac{n(r-1)+2}{n-2}$ and $\mu^{2}=\frac{n-2}{n(r-1)+2}$.

We consider the integral submanifold of the corresponding distribution of the space of principal vectors corresponding to the principal curvature $\lambda$. Since the multiplicity of the principal curvature $\lambda$ is greater than 1 , we know that the principal curvature $\lambda$ is constant on this integral submanifold (cf. Otsuki [6]). From $\lambda^{2}=\frac{n(r-1)+2}{n-2}$ and $\mu^{2}=\frac{n-2}{n(r-1)+2}$, we know that the scalar curvature $n(n-1) r$ and the principal curvature $\mu$ are constant. Thus, we obtain that $M$ is isoparametric. Therefore, $M$ is isometric to the Riemannian product $S^{1}\left(\sqrt{1-c^{2}}\right) \times S^{n-1}(c)$ because $S=(n-1) \frac{n(r-1)+2}{n-2}+$ $\frac{n-2}{n(r-1)+2}$ holds. This completes the Proof of Theorem 1 .

Proof of Theorem 2. Since the sectional curvatures are nonnegative, we have that the Ricci curvature is nonnegative. From the arguments in the 
Proof of Theorem 1, we infer that at each point, there exists a unit vector $\vec{u}$ such that $\operatorname{Ric}(\vec{u})=0$.

Let $\left\{e_{1}, \cdots, e_{n}\right\}$ be a local orthonormal frame field such that $h_{i j}=\lambda_{i} \delta_{i j}$, where $\lambda_{i}$ 's are principal curvatures on $M$. Then, from Gauss Equation (2.2), we have $1+\lambda_{i} \lambda_{j} \geq 0$ for $i \neq j$. Further, there exists an $i$ such that $\sum_{j \neq i}\left(1+\lambda_{i} \lambda_{j}\right)=0$ from the definition of Ricci curvature. Hence, we must have $1+\lambda_{i} \lambda_{j}=0$ for $j \neq i$. Therefore, $M$ has only two distinct principal curvatures one of which is simple. Let $\mu=\lambda_{i}$ and $\lambda=\lambda_{j}$ for $j \neq i$. From (2.4), we have

$$
\mu=\frac{n(r-1)}{2 \lambda}-\frac{n-2}{2} \lambda .
$$

Since $1+\mu \lambda=0$ and (2.13) hold, we have $\lambda^{2}=\frac{n(r-1)+2}{n-2}$ and $\mu^{2}=\frac{n-2}{n(r-1)+2}$. Hence, we have

$$
S=(n-1) \lambda^{2}+\mu^{2}=(n-1) \frac{n(r-1)+2}{n-2}+\frac{n-2}{n(r-1)+2} .
$$

By making use of the same assertion as in the Proof of Theorem 1, we infer that $M$ is isometric to the Riemannian product $S^{1}\left(\sqrt{1-c^{2}}\right) \times S^{n-1}(c)$. This completes the Proof of Theorem 2.

\section{References}

[1] T. Aubin, Some nonlinear problems in Riemannian geometry, Springer Monographs in Math., Springer-Verlag, Berlin, New York, 1998, MR 99i:58001, Zbl 0896.53003.

[2] Q.M. Cheng, Hypersurfaces in a unit sphere $S^{n+1}(1)$ with constant scalar curvature, J. London Math. Soc., 64 (2001), 755-768, MR 2002k:53116.

[3] Q.M. Cheng and H. Nakagawa, Totally umbilical hypersurfaces, Hiroshima Math. J., 20 (1990), 1-10.

[4] S.Y. Cheng and S.-T. Yau, Hypersurfaces with constant scalar curvature, Math. Ann., 225 (1977), 195-204, MR 55 \#4045, Zbl 0349.53041.

[5] H. Li, Hypersurfaces with constant scalar curvature in space forms, Math. Ann., 305 (1996), 665-672, MR 97i:53073, Zbl 0864.53040.

[6] T. Otsuki, Minimal hypersurfaces in a Riemannian manifold of constant curvature, Amer. J. Math., 92 (1970), 145-173, MR 41 \#9157, Zbl 0196.25102.

Received August 2, 2002. This research was partially supported by a Grant-in-Aid for Scientific Research from the Ministry of Education, Science and Culture, Japan.

Department of Mathematics

FaCUlty of Science And EngineEring

SAgA University, SAgA 840-8502

JAPAN

E-mail address: cheng@ms.saga-u.ac.jp 